\title{
Long-term progression-free survival of third-line apatinib in lung squamous cell carcinoma
}

This article was published in the following Dove Press journal:

OncoTargets and Therapy

\author{
Xiaofen $\mathrm{Li}^{1,2, *}$ \\ Xia $\mathrm{Li}^{3, *}$ \\ Xuefeng Fang' \\ Ying Yuan' \\ 'Department of Medical Oncology, \\ The Second Affiliated Hospital, \\ Zhejiang University School of \\ Medicine, Hangzhou, Zhejiang, China; \\ ${ }^{2}$ Department of Abdominal Oncology, \\ West China Hospital, Sichuan \\ University, Chengdu, Sichuan, China; \\ ${ }^{3}$ Department of Operation Room, \\ Zhejiang Province People's Hospital, \\ People's Hospital of Hangzhou \\ Medical College, Hangzhou, Zhejiang, \\ China \\ *These authors contributed equally \\ to this work
}

\begin{abstract}
To date, there is no standard third-line therapy for $E G F R$ - and $A L K$-negative lung squamous cell carcinoma (SCC). Apatinib, an oral small-molecule VEGFR-2 inhibitor, has shown good therapeutic effect and safety in chemotherapy-refractory gastric cancer, but its efficacy in lung SCC is not determined. In this report, we present a 69-year-old Chinese man with locally advanced EGFR- and ALK-negative lung SCC, who received apatinib after failure of 2 treatment regimens. After 4 months of apatinib therapy, chest computed tomography scan showed a large cavity in the lung tumor, while there was minimal change in tumor size. The patient had quite a long progression-free survival time of over 17 months and tolerated apatinib very well. Apatinib provides an additional option for salvage treatment of lung SCC without driver gene mutations, but further large-scale clinical studies are still warranted.
\end{abstract}

Keywords: apatinib, lung squamous cell carcinoma, efficacy

\section{Introduction}

Lung cancer is the leading cause of cancer-related death worldwide. ${ }^{1}$ Lung squamous cell carcinoma (SCC) accounts for about $30 \%$ of all lung cancer cases. Despite rapid progress in the treatment of non-small-cell lung cancer (NSCLC), there is no standard third-line therapy for $E G F R$ - and $A L K$-negative SCC patients. $^{2}$

Apatinib, an oral tyrosine kinase inhibitor (TKI) targeting VEGFR-2, shows efficacy as third-line treatment in metastatic gastric cancer and was approved by the China State Food and Drug Administration in December 2014. ${ }^{3-7}$ In 2012, a Phase II clinical study demonstrated that apatinib showed good efficacy as third-line treatment for nonsquamous NSCLC. ${ }^{8}$ However, there is limited data about the efficacy and toxicity of apatinib in the treatment of lung SCC so far. Herein, we report a patient with locally advanced lung SCC with wild-type EGFR and ALK gene expression who received apatinib after failure of 2 treatment regimens and obtained a long progression-free survival (PFS) of over 17 months.

\section{Ethics approval}

Written informed consent was provided by the patient to have the case details and any accompanying images published.

\section{Case presentation}

A 69-year-old Chinese man, who was a heavy smoker, was admitted to our hospital in August 2014, with a history of chest tightness and shortnes of breath on exertion lasting for two weeks. Enhanced chest computed tomography (CT) scan showed a $5 \times 6 \mathrm{~cm}$ mass in the right upper lobe with obstructive pneumonia and enlargement of right hilar 
and mediastinal lymph nodes. Pathologic results of bronchofibroscopic biopsy revealed lung SCC with right hilar and mediastinal lymph nodes metastases (Figure 1). Both of the $A L K$ and EGFR genes were wild type. Brain magnetic resonance imaging, bone emission CT, and abdominal ultrasound did not show any obvious distant metastasis.

Diagnosed with locally advanced lung SCC (cT3N2M0, stage IIIB), the patient received 6 cycles of first-line chemotherapy consisting of docetaxel $75 \mathrm{mg} / \mathrm{m}^{2}$ on day 1 and cisplatin $75 \mathrm{mg} / \mathrm{m}^{2}$ on day 1 , repeated every 3 weeks. Radiotherapy was given after 4 cycles of chemotherapy (primary lung cancer and mediastinum lymphoid region 6MV X-rays D95 PGTV 6450cGy/30F, D95 PTV 5400cGy/30F in 5 fields). According to Response Evaluation Criteria in Solid Tumors 1.1, the therapeutic evaluation was partial response after the second, fourth, and sixth cycles of chemotherapy. The patient tolerated treatment well and no severe adverse reaction occurred.

In July 2015, 5 months after the end of chemotherapy, chest CT scan revealed evident enlargement of the primary tumor in the right upper lobe and lymph nodes in the right hilum and mediastinum during a routine follow-up. The disease was evaluated as progressed, and so icotinib, an oral TKI, was prescribed as second-line therapy. Imaging examination indicated slight shrinkage of lung lesions and the patient was diagnosed as having stable disease during icotinib treatment.

Unfortunately, in March 2016, after 8 months of icotinib therapy, the disease progressed again (Figure 2A and $\mathrm{B}$ ). After discussion with the patient and his family, we prescribed apatinib (500 mg once a day) as third-line treatment. Imaging examination showed tumor shrinkage after 2 months of apatinib administration (Figure 2C). Moreover, about 4 months later, chest CT scan revealed a large cavity of $2.9 \times 1.6 \mathrm{~cm}$ in the lung tumor, with minimal change in tumor size (Figure 2D). Also, the obstructive pneumonia in the right lobe was significantly relieved. The latest chest CT scan on October 2017 showed a mass in the right lung hilum and mediastinum, so the disease had progressed once more
(Figure 2E). No severe adverse reaction was found during apatinib treatment. To date, for our patient, the PFS on thirdline apatinib has been longer than 6 months.

\section{Literature review and discussion}

Actually, antiangiogenic drugs have shown efficacy and have already been used in NSCLC, especially in patients without driver gene mutations. Early in 2006, the United States Food and Drug Administration approved bevacizumab for patients with unresectable, locally advanced, recurrent, or metastatic nonsquamous NSCLC. ${ }^{9,10}$ Recently, the Phase III study IFCT-1103 ULTIMATE, ${ }^{11}$ which compared the efficacy of paclitaxel with bevacizumab versus docetaxel as second- or third-line treatment for nonsquamous NSCLC, revealed that median PFS was longer in the paclitaxel plus bevacizumab arm compared with the docetaxel one (5.4 versus 3.9 months, hazard ratio [HR]: $0.62,95 \% \mathrm{CI}: 0.44-0.86, P=0.005)$. However, due to the risk of bleeding, bevacizumab is not recommended for SCC patients. Ramucirumab, a VEGFR-2 inhibitor, is another antiangiogenic agent approved by the US Food and Drug Administration for NSCLC. ${ }^{12}$ The Phase III trial, REVEL, ${ }^{13}$ indicated that compared with placebo, docetaxel with ramucirumab could prolong survival of advanced squamous or nonsquamous NSCLC patients who had failed platinum-based chemotherapy (median overall survival [OS]: 10.5 versus 9.1 months, HR: $0.86,95 \%$ CI: $0.75-0.98$, $P=0.023$; median PFS: 4.5 versus 3 months, HR: 0.76, 95\% CI: 0.68-0.86, $P<0.0001)$. Unfortunately, ramucirumab has not yet been approved for use in China.

Similarly, apatinib, an oral small-molecule VEGFR-2 inhibitor, is also an antiangiogenic agent. In December 2014, the China State Food and Drug Administration approved apatinib for advanced gastric patients who had failed at least 2 lines of chemotherapy. The randomized Phase III study of apatinib showed that, compared with placebo, apatinib could significantly improve OS and PFS in chemotherapy-refractory metastatic gastric cancer patients (median OS: 195 versus
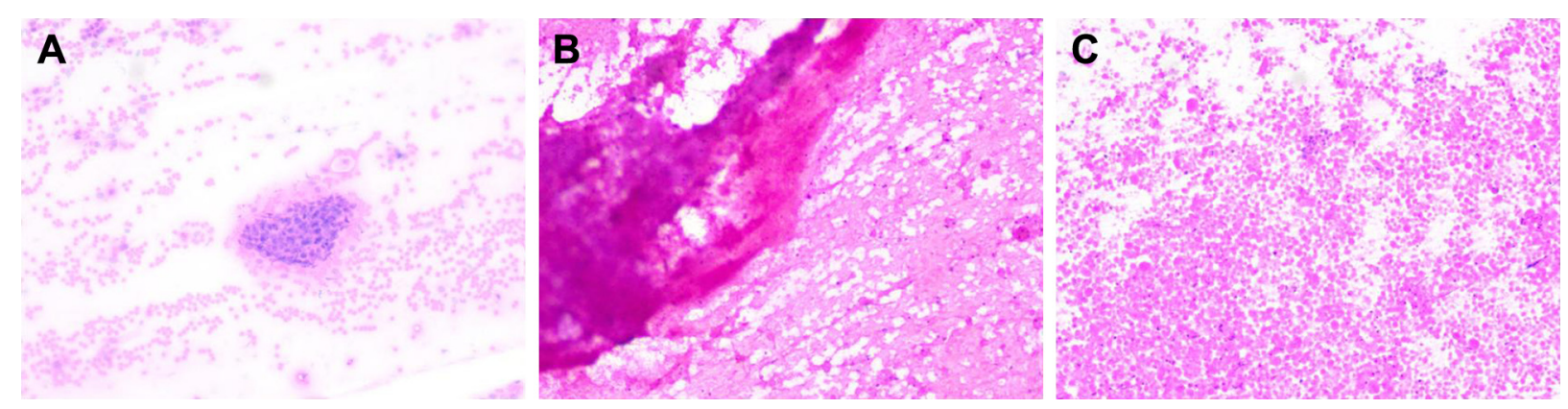

Figure I Bronchofibroscopic biopsy revealed SCC with opening of right upper lobar bronchus (A), and TBNA found cancer cells in 4R (B) and 7 lymph node groups (C). Abbreviations: SCC, squamous cell carcinoma; TBNA, transbronchial needle aspiration. 


\section{A}
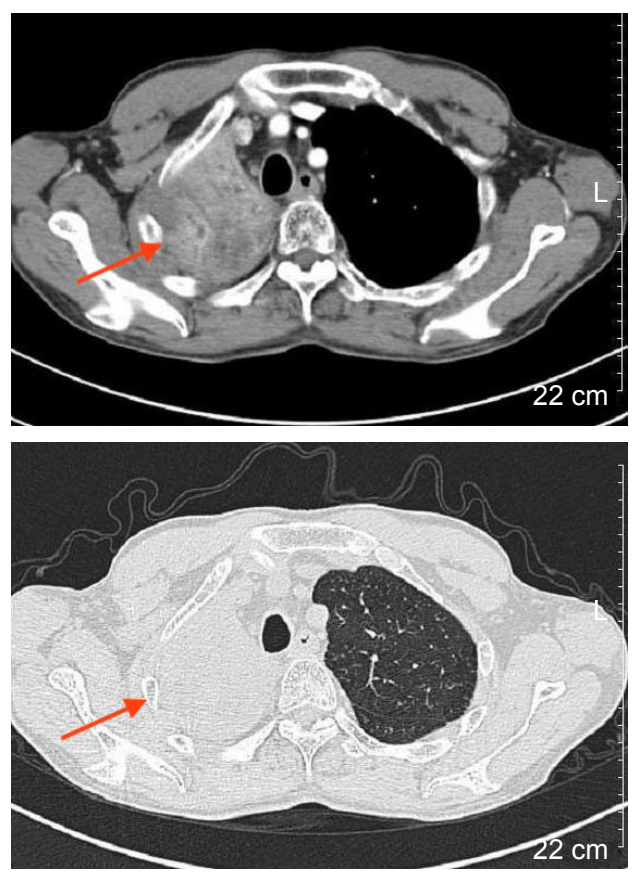

B

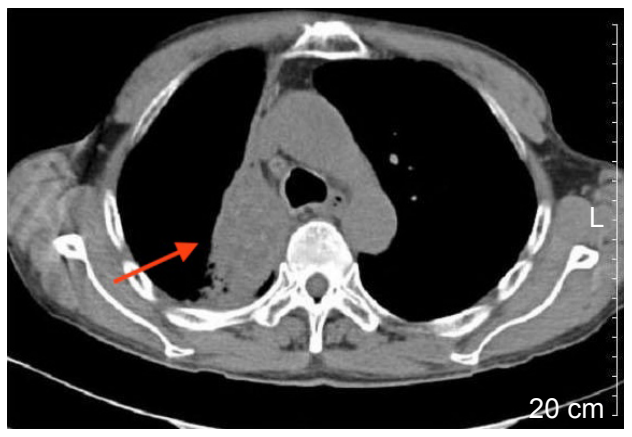

C
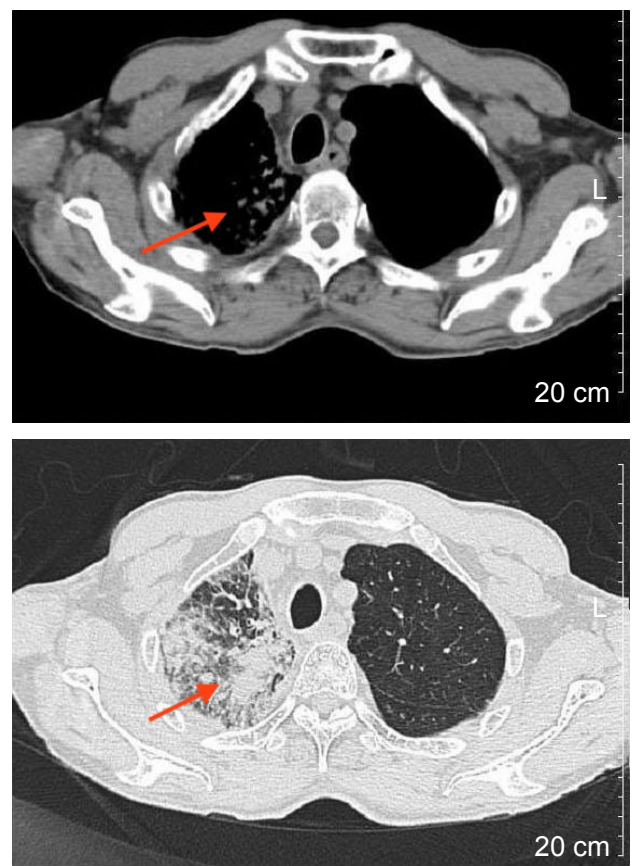

D

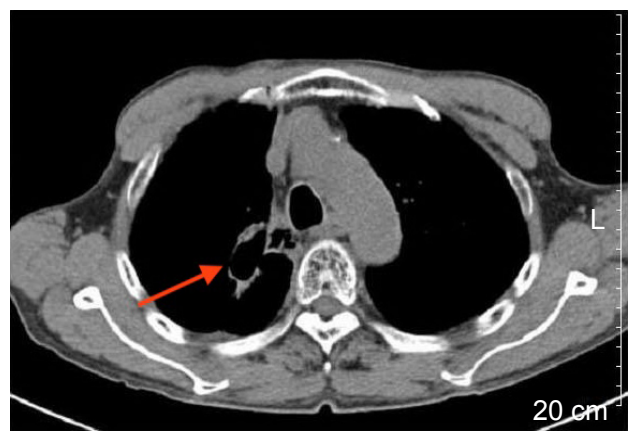

E

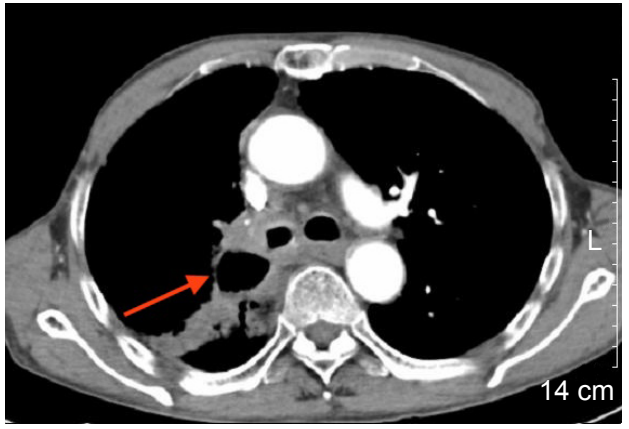

Figure 2 Chest CT images.

Notes: (A and B) the images shows after radiotheray and icotinib, that the right lung primary lesion and mediastinal lymph node were enlarged, and the disease had progressed in March 2016; (C) the image shows tumor shrinkage in May 2016; (D) the image reveals a large cavity in the lung tumor and minimal change in tumor size, in July 2016; (E) the image shows a mass in the right lung hilum and mediastinum, and disease progression once more in October 2017.

Abbreviation: CT, computed tomography.

140 days, HR: $0.71,95 \%$ CI: $0.54-0.94, P<0.016$; median PFS: 78 versus 53 days, HR: 0.44, 95\% CI: 0.33-0.61, $P<0.0001){ }^{3}$ The most common adverse events of apatinib were hypertension, hand-foot syndrome, and proteinuria, but the side effects were manageable and tolerable. . $^{3,4-6}$

Apart from gastric cancer, several studies have indicated that apatinib was effective in other solid tumors ${ }^{14}$ such as in breast cancer ${ }^{15,16}$ and NSCLC. ${ }^{8}$ A 1 -arm Phase II study ${ }^{14}$ which aimed to evaluate the optimum dose and efficacy of apatinib in heavily pretreated patients with metastatic triple-negative breast cancer concluded that the recommended dose of apatinib was $500 \mathrm{mg} / \mathrm{d}$, with encouraging median PFS (3.3 months, 95\% CI: 1.7-5.0) and OS (10.6 months, 95\% CI: 5.6-15.7).

Regarding use of apatinib in NSCLC, there has been only 1 Phase II study, ${ }^{8} 1$ retrospective study, ${ }^{17}$ and several case reports ${ }^{18-20}$ published so far. In the Phase II study, ${ }^{8}$ in patients 
with nonsquamous NSCLC who failed 2 lines of treatment, including EGFR TKIs, apatinib showed substantial clinical activity compared with placebo (median PFS: 4.7 versus 1.9 months, HR: $0.278,95 \%$ CI: $0.170-0.455, P<0.0001)$. In this literature review, adenocarcinoma is reported as the most common pathological type of lung cancer associated with apatinib application, apart from 1 retrospective study. This retrospective study ${ }^{16}$ enrolled a total of 42 advanced NSCLC patients including 12 with squamous carcinoma and 30 with adenocarcinoma, with a median PFS of 4.2 months and a median OS of 6 months.

One of the interesting aspects of our report is that the pathological type of cancer in our patient is advanced lung squamous carcinoma, regarding which details on the clinical efficacy and practical experience of apatinib are sorely lacking. Besides, the PFS of our patient was quite long at over 17 months. Previously reported PFS/median PFS in NSCLC patients using apatinib did not extend beyond 6 months. The reason for our patient showing such a long PFS is unknown, and needs further investigation. In summary, our report indicates that apatinib can provide an additional option for salvage treatment of lung SCC without driver gene mutations. However, further large-scale randomized case-control studies are warranted.

\section{Acknowledgment}

This paper was funded by Zhejiang Province Natural Science foundation of China (LY16H160027).

\section{Disclosure}

The authors report no conflicts of interest in this work.

\section{References}

1. Torre LA, Bray F, Siegel RL, Ferlay J, Lortet-Tieulent J, Jemal A. Global cancer statistics, 2012. CA Cancer J Clin. 2015;65(2):87-108.

2. Ettinger DS, Wood DE, Aisner DL, et al. Non-small cell lung cancer, version 5.2017, NCCN clinical practice guidelines in oncology. $J$ Natl Compr Canc Netw. 2017;15(4):504-535.

3. Qin S. Phase III study of apatinib in advanced gastric cancer: a randomized, double-blind, placebo-controlled trial. J Clin Oncol. 2014; 32(Suppl 15):4003.

4. Geng R, Li J. Apatinib for the treatment of gastric cancer. Expert Opin Pharmacother. 2015;16(1):117-122.

OncoTargets and Therapy

\section{Publish your work in this journal}

OncoTargets and Therapy is an international, peer-reviewed, open access journal focusing on the pathological basis of all cancers, potential targets for therapy and treatment protocols employed to improve the management of cancer patients. The journal also focuses on the impact of management programs and new therapeutic agents and protocols on
5. Tian S, Quan H, Xie C, et al. YN968D1 is a novel and selective inhibitor of vascular endothelial growth factor receptor-2 tyrosine kinase with potent activity in vitro and in vivo. Cancer Sci. 2011;102(7):1374-1380.

6. Li J, Qin S, Xu J, et al. Apatinib for chemotherapy-refractory advanced metastatic gastric cancer: results from a randomized, placebo-controlled, parallel-arm, phase II trial. J Clin Oncol. 2013;31(26):3219-3225.

7. Li J, Qin S, Xu J, et al. Randomized, double-blind, placebo-controlled Phase III trial of apatinib in patients with chemotherapy-refractory advanced or metastatic adenocarcinoma of the stomach or gastroesophageal junction. J Clin Oncol. 2016;34(13):1448-1454.

8. Zhang L, Shi M, Huang C, et al. A phase II, multicenter, placebocontrolled trial of apatinib in patients with advanced nonsquamous non-small cell lung cancer (NSCLC) after two previous treatment regimens. J Clin Oncol. 2012;30(Suppl 15):7548.

9. Cohen MH, Gootenberg J, Keegan P, Pazdur R. FDA drug approval summary: bevacizumab (Avastin) plus Carboplatin and Paclitaxel as first-line treatment of advanced/metastatic recurrent nonsquamous non-small cell lung cancer. Oncologist. 2007;12(6):713-718.

10. Sandler A, Gray R, Perry MC, et al. Paclitaxel-carboplatin alone or with bevacizumab for non-small-cell lung cancer. $N$ Engl J Med. 2006; 355(24):2542-2550.

11. Cortot AB, Audiger-Valette C, Molinier O, et al; French Cooperative Thoracic Intergroup (IFCT). Weekly paclitaxel plus bevacizumab versus docetaxel as second or third line treatment in advanced non-squamous non-small cell lung cancer (NSCLC): results from the phase III study IFCT-1103 ULTIMATE. J Clin Oncol. 2016;34(Suppl 15):9005.

12. Larkins E, Scepura B, Blumenthal GM, et al. U.S. Food and Drug Administration Approval Summary: ramucirumab for the treatment of metastatic non-small cell lung cancer following disease progression on or after platinum-based chemotherapy. Oncologist. 2015; 20(11):1320-1325.

13. Garon EB, Ciuleanu TE, Arrieta O, et al. Ramucirumab plus docetaxel versus placebo plus docetaxel for second-line treatment of stage IV non-small-cell lung cancer after disease progression on platinum-based therapy (REVEL): a multicentre, double-blind, randomised phase 3 trial. Lancet. 2014;384(9944):665-673.

14. Scott AJ, Messersmith WA, Jimeno A. Apatinib: a promising oral antiangiogenic agent in the treatment of multiple solid tumors. Drugs Today (Barc). 2015;51(4):223-229.

15. Hu X, Zhang J, Xu B, et al. Multicenter phase II study of apatinib, a novel VEGFR inhibitor in heavily pretreated patients with metastatic triple-negative breast cancer. Int J Cancer. 2014;135(8):1961-1969.

16. Hu X, Cao J, Hu W, et al. Multicenter phase II study of apatinib in nontriple-negative metastatic breast cancer. BMC Cancer. 2014;14:820.

17. Song Z, Yu X, Lou G, Shi X, Zhang Y. Salvage treatment with apatinib for advanced non-small-cell lung cancer. Onco Targets Ther. 2017;10: $1821-1825$.

18. Ding L, Li QJ, You KY, Jiang ZM, Yao HR. The use of apatinib in treating nonsmall-cell lung cancer: case report and review of literature. Medicine (Baltimore). 2016;95(20):e3598.

19. Fang SC, Zhang HT, Zhang YM, Xie WP. Apatinib as post second-line therapy in EGFR wild-type and ALK-negative advanced lung adenocarcinoma. Onco Targets Ther. 2017;10:447-452.

20. Peng Y, Cui H, Liu Z, et al. Apatinib to combat EGFR-TKI resistance in an advanced non-small cell lung cancer patient with unknown EGFR status: a case report. Onco Targets Ther. 2017;10:2289-2295.

patient perspectives such as quality of life, adherence and satisfaction The manuscript management system is completely online and includes a very quick and fair peer-review system, which is all easy to use. Visit http://www.dovepress.com/testimonials.php to read real quotes from published authors. 